\title{
INVOLUTIONS FIXING THE DISJOINT UNION OF 3-REAL PROJECTIVE SPACE WITH DOLD MANIFOLD*
}

\author{
ZhI LÜ AND XIBO LIU
}

\begin{abstract}
In this paper, we determine the existence of all involutions fixing a disjoint union of 3-real projective space $R P(3)$ with Dold manifold under the condition that the normal bundle to $R P(3)$ does not bound, and also study the representatives up to bordism of those involutions which exist.
\end{abstract}

\section{\$1. Introduction}

Let $(T, M)$ be an involution on a closed manifold, and let $F$ denote the fixed point set of $(T, M)$. When $F$ is chosen as

$$
\begin{gathered}
\{\mathrm{pt}\} \sqcup S^{m}, \quad R P(2 k), \quad R P(m) \sqcup R P(n), \quad \sqcup R P(2 l+1)(l \text { fixed }), \\
\sqcup_{i=1}^{p} R P\left(2 l_{i}+1\right), \quad \sqcup_{i=1}^{r}\left(S^{1}\right)^{k_{l}},
\end{gathered}
$$

and $\left(S^{n_{1}} \times S^{n_{2}} \times \cdots \times S^{n_{p}}\right) \sqcup\{\mathrm{pt}\}$, respectively, the existence and the representative (up to bordism) of $(T, M)$ have been studied in [2], [11], [9], [13], [4], [12] and [8]. The purpose of this paper is to determine the existence and the representative up to bordism of all involutions fixing a disjoint union $R P(3) \sqcup P(m, n)$, where $P(m, n)$ is the Dold manifold of dimension $m+2 n$ obtained from the product $S^{m} \times C P(n)$ of the $m$-sphere with the $n$-dimensional complex projective space by identifying $(x, z)$ with $(-x, \bar{z})$ (here $(x, z) \in S^{m} \times C P(n)$ ). For this purpose, we first study the vector bundle over Dold manifold so that we can begin with our discussion on the existence of all involutions. The main method will be a formula given by Kosniowski and Stong in [5], and Lucas Theorem [10] will also be used. By setting an involution on Dold manifold $P(3, n+1)$, we partially give the representatives up to bordism of those involutions which exist.

1991 Mathematics Subject Classification: 57R85, 57R90, 55N22.

Keywords: Involution, Dold manifold, symmetric polynomial function, characteristıc class, bordism.

* This work is supported by the Youthful Foundation of Tsinghua University, and partly by the Japanese Government Sholarship.

Recelved October 20, 1998; revised April 28, 1999. 
In particular, we will see that the case $F=R P(3) \sqcup P(m, n)$ possesses more complicated structure than those cases in [2], [4], [8], [9], [11], [12] and [13].

Throughout this paper, the coefficient group is $Z_{2}$. Let $\omega$ be the total Stiefel-Whitney class and $\omega_{i}$ the $i$-th Stiefel-Whitney class. Let $[N]$ denote the fundamental homology class of manifold $N$. Let $\sigma_{i}(x)$ denote the $i$-th elementary symmetric function $\Sigma x_{1} \cdots x_{l}$.

The authors wish to express their gratitude to Professors Takashi Tsuboi, Zhende $\mathrm{Wu}$, and Zongze Liu for their helps and encouragements during the preparation of this work, and also to the referee for his valuable suggestions.

\section{§2. The total Stiefel-Whitney class of vector bundle over Dold manifold $P(m, n)$}

Following the notation of [14], let $\xi$ be a 1-plane bundle over $P(m, n)$ and $\eta$ a 2-plane bundle over $P(m, n)$. Let $c \in H^{1}\left(P(m, n) ; Z_{2}\right)$ be the generator and $d \in H^{2}\left(P(m, n) ; Z_{2}\right)$ the generator. Then $\omega \xi=1+c, \omega \eta=1+c+d, \xi \otimes \xi=1$, $\xi \otimes \eta=\eta$ (see [14; Proposition (1.4)]).

LEMMA 2.1. Let $\tau$ be the tangant bundle over $P(m, n)$. Then $\omega \tau=$ $(1+c)^{m}(1+c+d)^{n+1}$, also denoted by $\omega P(m, n)$.

Proof. From [14; Theorem (1.5)] we know

$$
\tau \oplus \xi \oplus 2=(m+1) \xi \oplus(n+1) \eta .
$$

Hence it immediately follows that $\omega \tau=(1+c)^{m}(1+c+d)^{n+1}$.

Let $\varphi(l)=$ the number of integers $e$ with $0<e \leq l$ and $e \equiv 0,1,2,4(\bmod 8)$.

LeMMA 2.2. Let $\lambda$ be any a vector bundle over $P(m, n)$. If $n$ is even and $m \equiv 0,1,3,7(\bmod 8)$, then

$$
\omega \lambda=(1+c)^{s}(1+c+d)^{t}
$$

where $s$ and $t$ are nonnegative integers.

Proof. Let $\alpha=\xi-1, \beta=\eta-2, \gamma=\beta-\alpha$. According to [3; Theorem 5], when $n$ is even and $m \equiv 0,1,3,7(\bmod 8)$, we know that $\tilde{K} O(P(m, n))$ is isomorphic to $Z_{2^{\varphi(m)}}+Z^{n / 2}$ generated by $\alpha, \gamma, \gamma^{2}, \ldots, \gamma^{n / 2}$ such that $2^{\varphi(m)} \alpha=0$, $\alpha^{2}=-2 \alpha$ and $\alpha \gamma=0$. Therefore it suffices to compute $\omega \xi$ and $\omega \underbrace{(\eta \otimes \cdots \otimes \eta)}_{p}$ for all $p \geq 0$. Using the formula in [7; p. 87] and $\omega \eta=1+c+d$, by direct computions, we can obtain that

$$
\omega \underbrace{(\eta \otimes \cdots \otimes \eta)}_{2 l}=(1+c)^{2^{2 l-1}}
$$


and

$$
\omega \underbrace{(\eta \otimes \cdots \otimes \eta)}_{2 l+1}=(1+c+d)^{2^{2 l}} .
$$

Again since $\omega \xi=1+c$, it follows that $\omega \lambda$ can be as stated.

\section{§3. A formula and Lucas Theorem}

In this section, we review a formula given by Kosniowski and Stong and Lucas Theorem, which will play the important roles in the following sections.

Let $\left(T, M^{n}\right)$ be a smooth involution on a closed $n$-manifold and the fixed point data of $\left(T, M^{n}\right)$ be $\mu \rightarrow F=\sqcup_{k} \mu^{k} \rightarrow F^{n-k}$. In [5], Kosniowski and Stong gave a formula for the calculation of the Stiefel-Whitney numbers of $M^{n}$ in terms of the fixed point data $\mu \rightarrow F=\sqcup_{k} \mu^{k} \rightarrow F^{n-k}$. That is the following

THEOREM 3.1 (Kosniowski and Stong). If $f\left(x_{1}, \ldots, x_{n}\right)$ is any symmetric polynomial over $Z_{2}$ in $n$ variables of degree at most $n$, then

$$
f\left(x_{1}, \ldots, x_{n}\right)\left[M^{n}\right]=\sum_{k} \frac{f\left(1+y_{1}, \ldots, 1+y_{k}, z_{1}, \ldots, z_{n-k}\right)}{\prod_{l=1}^{k}\left(1+y_{i}\right)}\left[F^{n-k}\right]
$$

where the expressions are evaluated by replacing the elementary symmetric functions $\sigma_{i}(x), \sigma_{i}(y)$, and $\sigma_{i}(z)$ by the Stiefel-Whitney classes $\omega_{i}\left(M^{n}\right), \omega_{i}\left(\mu^{k}\right)$, and $\omega_{i}\left(F^{n-k}\right)$ respectively, and taking the value of the resulting cohomology class on the fundamental homology class of $M^{n}$ or $F^{n-k}$.

Besides, in their paper [5], Kosniowski and Stong also obtained the following

Proposition 3.2. Let $\sqcup_{k} \mu^{k} \rightarrow F^{n-k}$ be a disjoint union of bundles over manifolds. A necessary and sufficient condition that the disjoint union is the fixed point data of an involution $\left(T, M^{n}\right)$ is that

$$
\sum_{k} \frac{f(1+y, z)}{\prod(1+y)}\left[F^{n-k}\right]=0
$$

for all $f$ of degree less than $n$.

By Proposition 3.2, it is easy to see the following result.

COROLlaRY 3.3. Let $\sqcup_{k} \mu^{k} \rightarrow F^{n-k}$ be a disjoint union of bundles over manifolds. For some positive integer $m$, if $\sqcup_{k} \mu^{k} \oplus m R \rightarrow F^{n-k}$ is the fixed point data of some involution, then so is $\sqcup_{k} \mu^{k} \oplus i R \rightarrow F^{n-k}$ for each $i<m$.

Given any involution $\left(T, M^{n}\right)$, as defined in [1], let $\Gamma^{1}\left(M^{n}\right)$ denote a $(n+1)$ manifold obtained from $S^{1} \times M^{n}$ by identifying $(z, x)$ with $(-z, T x)$, and with 
an involution $T_{1}$ on $\Gamma^{1}\left(M^{n}\right)$ induced by $(z, x) \rightarrow(\bar{z}, x)$. Let $\left(T_{0}, \Gamma^{0}\left(M^{n}\right)\right)=$ $\left(T, M^{n}\right)$, and let $\left(T_{l}, \Gamma^{l}\left(M^{n}\right)\right)$ be the $i$-th iteration of $\left(T, M^{n}\right)$. Then a sequence of involutions $\left\{\left(T_{l}, \Gamma^{l}\left(M^{n}\right)\right)\right\}$ are constructed. In addition, we also know from [1] that the normal bundle to the fixed point set of $\left(T_{l}, \Gamma^{l}\left(M^{n}\right)\right)$ is

$$
\sqcup_{k}\left(\mu^{k} \oplus i R \rightarrow F^{n-k}\right) \sqcup\left(\sqcup_{j=0}^{l-1}(i-j) R \rightarrow \Gamma^{J}\left(M^{n}\right)\right)
$$

where $\mu \rightarrow F=\sqcup_{k}\left(\mu^{k}-F^{n-k}\right)$ is the original normal bundle to $F$ in $M^{n}$. The following lemmas will be used in $\$ \$ 5,6$.

Lemma 3.4 (See [6]). Let $\mu \rightarrow F=\sqcup_{k}\left(\mu^{k} \rightarrow F^{n-k}\right)$ be the fixed data of an involution $\left(T, M^{n}\right)$. Then a necessary and sufficient condition that $\mu \oplus m R \rightarrow F$ is the fixed data of some involution is that $\Gamma^{J}\left(M^{n}\right)$ bounds for each $j<m$.

LEMMA 3.5. Let $\left(T, M^{n}\right)$ be an involution and its fixed data be $\mu \rightarrow$ $F=\sqcup_{k} \mu^{k} \rightarrow F^{n-k}$. If $\mu \oplus i R \rightarrow F$ is the fixed data of some involution, denoted by $\left(T^{\prime}, M^{\prime}\right)$, then $\left(T^{\prime}, M^{\prime}\right)$ is bordant to $\left(T_{l}, \Gamma^{l}\left(M^{n}\right)\right)$.

Proof. First, by using Proposition 3.2, it is easy to show that $\sqcup_{j=0}^{l-1}(i-j) R$ $\left.\rightarrow \Gamma^{J}\left(M^{n}\right)\right)$ is still the fixed data of some involution. Next, by [2; Theorem (23.1)], it immediately follows that $\Gamma^{J}\left(M^{n}\right)$ bounds for $0 \leq j \leq i-1$, and thus $\sqcup_{k} \mu^{k} \oplus i R \rightarrow F^{n-k}$ is bordant to $\left(\sqcup_{k} \mu^{k} \oplus i R \rightarrow F^{n-k}\right) \sqcup\left(\sqcup_{j=0}^{l-1}(i-j) R \rightarrow \Gamma^{J}\left(M^{n}\right)\right)$. Furthermore, by [2; Theorem (25.2)], we obtain that $\left(T^{\prime}, M^{\prime}\right)$ is bordant to $\left(T_{l}, \Gamma^{l}\left(M^{n}\right)\right)$. This completes the proof.

For any positive integer $l$, let $E(l)$ denote a set formed by all $i_{1}, \ldots, i_{\alpha}$ in $2^{l_{1}}+\cdots+2^{l_{\alpha}}$, where $2^{l_{1}}+\cdots+2^{l_{\alpha}}$ is the dyadic decomposition of $l$. Then we have

ThEOREM 3.6 (Lucas) (see [10]). Let $p, q$ be two positive integers. Then $\left(\begin{array}{l}p \\ q\end{array}\right) \equiv 1(\bmod 2)$ if and only if $E(q) \subset E(p)$.

\section{§4. The cases in which involutions do not exist}

Throughout the following sections, we always suppose that $\left(T, M^{m+2 n+k}\right)$ is an involution on a closed $(m+2 n+k)$-manifold with fixed point set $F=$ $R P(3) \sqcup P(m, n)$ where $m, n>0$. Let $v \rightarrow F=v_{1} \rightarrow R P(3) \sqcup v_{2} \rightarrow P(m, n)$ be the normal bundle of $F$ in $M^{m+2 n+k}$. First, by [2], [9] and [13], we have $\omega R P(3)=$ $(1+a)^{4}=1$ and $\omega v_{1}=(1+a)^{h}$ where $h$ is nonnegative integer and $a \in$ $H^{1}\left(R P(3) ; Z_{2}\right)$ is the generator. Let $c \in H^{1}\left(P(m, n) ; Z_{2}\right)$ be the generator and let $d \in H^{2}\left(P(m, n) ; Z_{2}\right)$ be the generator, by Lemma 2.1 and Lemma 2.2 , it follows that $\omega P(m, n)=(1+c)^{m}(1+c+d)^{n+1}$ and for $m \equiv 0,1,3,7(\bmod 8)$ and even $n, \omega v_{2}=(1+c)^{s}(1+c+d)^{t}$.

Notice that if $v_{1} \rightarrow R P(3)$ bounds, i.e., $\omega v_{1}=1$ or $(1+a)^{2}$, then $v_{1} \rightarrow$ $R P(3) \sqcup v_{2} \rightarrow P(m, n)$ is bordant to $v_{2} \rightarrow P(m, n)$. By [2; Theorem (25.2)], this 
means that the case $F=R P(3) \sqcup P(m, n)$ is identied with the case $F=P(m, n)$. Here we focus our attention on the case $F=R P(3) \sqcup P(m, n)$ with nonbounding normal bundle $v_{1} \rightarrow R P(3)$. Hence in the following discussions, we always suppose that $v_{1} \rightarrow R P(3)$ does not bound, i.e., $\omega v_{1}=1+a$ or $(1+a)^{3}$.

Now we begin on discussing the existence of $\left(T, M^{m+2 n+k}\right)$.

(I) The case $\chi(P(m, n))=1$, where $\chi($.$) denotes the Euler characteristic$ number.

LEMMA 4.1. There does not exist the involution $\left(T, M^{m+2 n+k}\right)$ with $\chi(P(m, n))=1$.

Proof. Suppose that $\left(T, M^{m+2 n+k}\right)$ exists if $\chi(P(m, n))=1$. First, by [5; p. 313, Proposition], we have

$$
\chi\left(M^{m+2 n+k}\right)=\chi(R P(3))+\chi(P(m, n))=0+1=1 .
$$

Hence it immedately follows that $m, n, k$ must be all even. Next, using the fact (see $[5 ;$ p. 317 , Lemma]) that

$$
\begin{aligned}
\sigma_{i}\left(1+y_{1}, \ldots, 1+y_{e}, z_{1}, \ldots, z_{n-e}\right) \\
\quad=\sum_{p+q \leq l}\left(\begin{array}{c}
e-p \\
i-p-q
\end{array}\right) \sigma_{p}\left(y_{1}, \ldots, y_{e}\right) \sigma_{q}\left(z_{1}, \ldots, z_{n-e}\right),
\end{aligned}
$$

by direct computations, we have

$$
\sigma_{1}(1+y, z)\left(v_{1} \rightarrow R P(3)\right)=1+a
$$

and

$$
\sigma_{1}(1+y, z)\left(v_{2} \rightarrow P(m, n)\right)=\varepsilon c
$$

where $\varepsilon=0$ or 1 . We proceed as follows:

Case (i) When $\omega v_{1}=1+a$, if $m=4 l+2$, taking $f(x)=\left(\sigma_{1}(x)\right)^{m+2}$. $(\operatorname{deg} f=m+2=4 l+4<m+2 n+k)$ and using Theorem 3.1, then we have

$$
\begin{aligned}
0 & =f(x)\left[M^{m+2 n+k}\right] \\
& =\frac{\left(\sigma_{1}(1+y, z)\right)^{4 l+4}}{\prod(1+y)}[R P(3)]+\frac{\left(\sigma_{1}(1+y, z)\right)^{4 l+4}}{\prod(1+y)}[P(4 l+2, n)] \\
& =\frac{(1+a)^{4 l+4}}{1+a}[R P(3)]+\frac{(\varepsilon c)^{4 l+4}}{\omega v_{2}}[P(4 l+2, n)] \\
& =\left(1+a+a^{2}+a^{3}\right)[R P(3)]+0 \\
& =1 .
\end{aligned}
$$

This leads to a contradiction. If $m=4 l$, first it is easy to see that $k$ must be a positive even. Taking $\left(\sigma_{1}(x)\right)^{m+4}$ of degree less than $m+2 n+k$ and using 
Theorem 3.1, we have

$$
\begin{aligned}
0 & =\frac{(1+a)^{4 l+4}}{1+a}[R P(3)]+\frac{(\varepsilon c)^{4 l+4}}{\omega v_{2}}[P(4 l, n)] \\
& =1+0 \\
& =1
\end{aligned}
$$

But this is impossible.

Case (ii) When $\omega v_{1}=(1+a)^{3}$, if $m=4 l+2$ then we choose $\left(\sigma_{1}(x)\right)^{m+4}$ and if $m=4 l$ then we choose $\left(\sigma_{1}(x)\right)^{m+2}$, as in the case (i), we can obtain the contradictions and thus the case $\omega v_{1}=(1+a)^{3}$ doesn't exist.

Combining the above discussions, the Lemma thus holds.

(II) The case $\chi(P(m, n))=0$.

From $\chi(P(m, n))=0$, we have $(m+1)(n+1) \equiv 0(\bmod 2)$. Therefore, in the following discussions we divide $\chi(P(m, n))=0$ into three cases: (i) $m$ is even and $n$ is odd; (ii) $m, n$ are all odd; (iii) $m$ is odd and $n$ is even.

Notice that when $k=0$, i.e., $\omega v_{2}=1$, it is easy to see that $\left(T, M^{m+2 n+k}\right)$ with $\chi(P(m, n))=0$ does not exist. Hence here we may assume that $k>0$.

LEMma 4.2. There does not exist the involution $\left(T, M^{m+2 n+k}\right)$ for which $m$ is even and $n$ is odd.

Proof. Suppose that when $m$ is even and $n$ is odd, the involution $\left(T, M^{m+2 n+k}\right)$ exists. We proceed as follows:

If $\omega v_{1}=1+a$, using [5; p. 317 , Lemma], then we have

$$
\sigma_{1}(1+y, z)\left(v_{1} \rightarrow R P(3)\right)=\left(\begin{array}{c}
m+2 n+k-3 \\
1
\end{array}\right)+a
$$

and

$$
\sigma_{1}(1+y, z)\left(v_{2} \rightarrow P(m, n)\right)=\left(\begin{array}{c}
k \\
1
\end{array}\right)+\varepsilon c
$$

where $\varepsilon=0$ or 1 . Consider the following:

(i) When $m=4 l+2$, taking $\left(\sigma_{1}(x)+\left(\begin{array}{l}k \\ 1\end{array}\right)\right)^{m+2}$ of degree less than $m+2 n+k$ and using Theorem 3.1, we have

$$
0=\frac{(1+a)^{4 l+4}}{1+a}[R P(3)]+\frac{(\varepsilon c)^{4 l+4}}{\omega v_{2}}[P(4 l+2, n)]=1+0=1
$$

This means that $m=4 l+2$ is impossible. 
(ii) When either $m=4 l, n>1$ or $m=4 l, n=1, k>2$, taking $\left(\sigma_{1}(x)+\right.$ $\left.\left(\begin{array}{l}k \\ 1\end{array}\right)\right)^{m+4}$, in the same way as the above, we can show that either $m=4 l, n>1$ or $m=4 l, n=1, k>2$ is impossible. have

(iii) When $m=4 l, n=1, k=1$, taking symmetric polynoimal function 1 , we

$$
0=\frac{1}{1+a}[R P(3)]+\frac{1}{1+\sigma_{1}(y)}[P(4 l, 1)]=1+0=1 .
$$

But this is a contradiction.

(iv) When $m=4 l, n=1, k=2$, if $\omega_{1} v_{2}=0$, i.e., $\sigma_{1}(1+y, z)\left(v_{2} \rightarrow P(m, n)\right)$ $=\left(\begin{array}{l}k \\ 1\end{array}\right)=0$, taking $\left(\sigma_{1}(x)\right)^{4 l}$, then we can obtain a contradiction, and thus $\omega_{1} v_{2}=$ 0 is impossible. Let $\omega_{1} v_{2}=c$. Using [5; p. 317, Lemma], we have

$$
\sigma_{2}(1+y, z)\left(v_{1} \rightarrow R P(3)\right)=0
$$

and

$$
\sigma_{2}(1+y, z)\left(v_{2} \rightarrow P(4 l, 1)\right)=1+c+c^{2}+\sigma_{2}(y) .
$$

Taking $\left(\sigma_{2}(x)+\sigma_{1}^{2}(x)\right)^{2}$ and using Theorem 3.1, it follows that

$$
\begin{aligned}
0 & =\frac{(1+a)^{4}}{1+a}[R P(3)]+\frac{\left(1+c+\sigma_{2}(y)\right)^{2}}{1+c+\sigma_{2}(y)}[P(4 l, 1)] \\
& =1+\left(1+c+\sigma_{2}(y)\right)[P(4 l, 1)] \\
& =1+0 \\
& =1 .
\end{aligned}
$$

But this is a contradiction.

If $\omega v_{1}(1+a)^{3}$, in the same way as the case $\omega v_{1}=1+a$, take $\left(\sigma_{1}(x)+\right.$ $\left.\left(\begin{array}{l}k \\ 1\end{array}\right)\right)^{m+2}$, we may prove that $m=4 l$ is impossible; and take $\left(\sigma_{1}(x)+\left(\begin{array}{l}k \\ 1\end{array}\right)\right)^{m+4}$, we may prove that either $m=4 l+2, n>1$ or $m=4 l+2, n=1, k>2$ is impossible. When $m=4 l+2, n=1$, and $k=1$, taking $\left(\sigma_{1}(x)\right)^{2}$, we have

$$
\begin{aligned}
0 & =\frac{a^{2}}{(1+a)^{3}}[R P(3)]+\frac{\left(1+\sigma_{1}(y)\right)^{2}}{1+\sigma_{1}(y)}[P(4 l+2,1)] \\
& =1+\left(1+\sigma_{1}(y)\right)[P(4 l+2,1)] \\
& =1+0 \\
& =1 .
\end{aligned}
$$

So this case is impossible. When $m=4 l+2, n=1$, and $k=2$, since

$$
\sigma_{2}(1+y, z)\left(v_{1} \rightarrow R P(3)\right)=1+a^{2}
$$

and

$$
\sigma_{2}(1+y, z)\left(v_{2} \rightarrow P(4 l, 1)\right)=1+\sigma_{1}(y)+\sigma_{2}(y)
$$


choose $\sigma_{2}(x)$, it follows that

$$
\begin{aligned}
0 & =\frac{1+a^{2}}{(1+a)^{3}}[R P(3)]+\frac{1+\sigma_{1}(y)+\sigma_{2}(y)}{1++\sigma_{1}(y)+\sigma_{2}(y)}[P(4 l+2,1)] \\
& =1+[P(4 l+2,1)] \\
& =1+0 \\
& =1 .
\end{aligned}
$$

This means that the case in which $m=4 l+2, n=1$, and $k=2$ is still impossible.

Together with the above discussions, we complete the proof of the Lemma 4.2.

LEMMA 4.3. There does not exist the involution $\left(T, M^{m+2 n+k}\right)$ for which $m, n$ are all odd.

Proof. Suppose that when $m, n$ are all odd, the involution $\left(T, M^{m+2 n+k}\right)$ exists. Using $[5 ;$ p. 317 , Lemma $]$, we have

$$
\sigma_{1}(1+y, z)\left(v_{1} \rightarrow R P(3)\right)=\left(\begin{array}{c}
m+2 n+k-3 \\
1
\end{array}\right)+a
$$

and

$$
\sigma_{1}(1+y, z)\left(v_{2} \rightarrow P(m, n)\right)=\left(\begin{array}{c}
k \\
1
\end{array}\right)+c+\sigma_{1}(y) .
$$

If either $\omega_{1} v_{2}=c$ (i.e., $\left.\sigma_{1}(1+y, z)\left(v_{2} \rightarrow P(m, n)\right)=\left(\begin{array}{l}k \\ 1\end{array}\right)\right)$ or $m=1$, taking $\left(\sigma_{1}(x)+\left(\begin{array}{l}k \\ 1\end{array}\right)\right)^{3}$ and using Theorem 3.1, then

$$
0=\frac{a^{3}}{\omega v_{1}}[R P(3)]+\frac{0}{\omega v_{2}}[P(m, n)]=1+0=1
$$

But this is a contradiction. If $\omega_{1} v_{2}=0$, by direct computations, we have $\sigma_{2}(1+y, z)\left(v_{1} \rightarrow R P(3)\right)=\left(\begin{array}{c}m+2 n+k-3 \\ 2\end{array}\right)+\left(\begin{array}{c}m+2 n+k-4 \\ 1\end{array}\right) \sigma_{1}(y)+\sigma_{2}(y)$ and

$$
\sigma_{2}(1+y, z)\left(v_{2} \rightarrow P(m, n)\right)=\left(\begin{array}{l}
k \\
2
\end{array}\right)+\left(\begin{array}{l}
k \\
1
\end{array}\right) c+\sigma_{2}(y)+\left(\begin{array}{c}
m+n+1 \\
2
\end{array}\right) c^{2} .
$$

We proceed as follows: taking

(i) When $\sigma_{2}(y)+\left(\begin{array}{c}m+n+1 \\ 2\end{array}\right) c^{2}=\varepsilon c^{2}+d$ (here $\varepsilon=0$ or 1) in (4.1) and $m \neq 3$,

$$
f(x)=\left(\sigma_{1}(x)+\left(\begin{array}{l}
k \\
1
\end{array}\right)\right)^{m}\left(\sigma_{2}(x)+\left(\begin{array}{l}
k \\
2
\end{array}\right)+\left(\begin{array}{c}
k-1 \\
1
\end{array}\right)\left(\sigma_{1}(x)+\left(\begin{array}{l}
k \\
1
\end{array}\right)\right)\right)^{n}
$$


with $\operatorname{deg} f=m+2 n<m+2 n+k$, we have

but

$$
\begin{aligned}
0 & =\frac{f(1+y, z)}{\omega v_{1}}[R P(3)]+\frac{c^{m}\left(c+\varepsilon c^{2}+d\right)^{n}}{\omega v_{2}}[P(m, n)] \\
& =\frac{f(1+y, z)}{\omega v_{1}}[R P(3)]+1,
\end{aligned}
$$

$$
\begin{aligned}
& \frac{f(1+y, z)}{\omega v_{1}}[R P(3)] \\
& \quad= \begin{cases}\frac{a^{m}\left(\sigma_{2}(y)\right)^{n}}{\omega v_{1}}[R P(3)]=0, & \text { if } m \equiv 1,5(\bmod 8)(m \neq 1) ; \\
\frac{a^{m}\left(1+\sigma_{2}(y)\right)^{n}}{\omega v_{1}}[R P(3)]=0, & \text { if } m \equiv 3,7(\bmod 8)(m \neq 3) .\end{cases}
\end{aligned}
$$

Obviously, this leads to a contradiction.

(ii) When $\sigma_{2}(y)+\left(\begin{array}{c}m+n+1 \\ 2\end{array}\right) c^{2}=\varepsilon c^{2}+d$ (here $\left.\varepsilon \in Z_{2}\right)$ in (4.1) and $m=3$, it is obvious that $k$ must be more than or equal to 2 . If $k>2$, taking

$$
\left(\sigma_{1}(x)+\left(\begin{array}{l}
k \\
1
\end{array}\right)\right)^{3}\left(\sigma_{2}(x)+\left(\begin{array}{l}
k \\
2
\end{array}\right)+\left(\begin{array}{c}
k-1 \\
1
\end{array}\right)\left(\sigma_{1}(x)+\left(\begin{array}{c}
k \\
1
\end{array}\right)\right)\right)^{n+1}
$$

of degree less than $m+2 n+k$, we can obtain the following contrary equation

$$
\begin{aligned}
0 & =\frac{a^{3}\left(1+\sigma_{2}(y)\right)^{n+1}}{\omega v_{1}}[R P(3)]+\frac{c^{3}\left(c+\varepsilon c^{2}+d\right)^{n+1}}{\omega v_{2}}[P(3, n)] \\
& =1+0 \\
& =1
\end{aligned}
$$

Note that $\left(\begin{array}{l}k \\ 2\end{array}\right)+\left(\begin{array}{c}2 n+k \\ 2\end{array}\right)=1$. For $k=2$, if $\omega v_{1}=1+a$, taking $\left(1+\sigma_{1}(x)\right)^{4}$, then we have the following contrary equation

$$
0=\frac{(1+a)^{4}}{1+a}[R P(3)]+\frac{(1+c)^{4}}{1+\left(\begin{array}{c}
4+n \\
2
\end{array}\right) c^{2}+\varepsilon c^{2}+d}[P(3, n)]=1+0=1
$$

if $\omega v_{1}=(1+a)^{3}$, taking $\left(1+\sigma_{1}(x)\right)^{2}$, then we have

$$
0=\frac{(1+a)^{2}}{(1+a)^{3}}[R P(3)]+\frac{(1+c)^{2}}{1+\left(\begin{array}{c}
4+n \\
2
\end{array}\right) c^{2}+\varepsilon c^{2}+d}[P(3, n)]=1+0=1,
$$

but this is also a contradiction.

(iii) When $\sigma_{2}(y)+\left(\begin{array}{c}m+n+1 \\ 2\end{array}\right) c^{2}=\varepsilon c^{2}$ in $(4.1)$, if $m \equiv 1,5(\bmod 8)(m \neq 1)$, taking $\left(\sigma_{1}(x)+\left(\begin{array}{l}k \\ 1\end{array}\right)\right)^{2}\left(\sigma_{2}(x)+\left(\begin{array}{c}k \\ 2\end{array}\right)+\left(\begin{array}{c}k-1 \\ 1\end{array}\right)\left(\sigma_{1}(x)+\left(\begin{array}{l}k \\ 1\end{array}\right)\right)\right)+\left(\sigma_{1}(x)+\left(\begin{array}{l}k \\ 1\end{array}\right)\right)^{3}+\varepsilon\left(\sigma_{1}(x)\right.$ 
$\left.+\left(\begin{array}{l}k \\ 1\end{array}\right)\right)^{4}$, we have

$$
\begin{aligned}
0 & =\frac{a^{2} \sigma_{2}(y)+a^{3}+\varepsilon a^{4}}{\omega v_{1}}[R P(3)]+\frac{c^{2}\left(c+\varepsilon c^{2}\right)+c^{3}+\varepsilon c^{4}}{1+\left(\begin{array}{c}
m+n+1 \\
2
\end{array}\right) c^{2}+\varepsilon c^{2}}[P(m, n)] \\
& =1+0 \\
& =1 .
\end{aligned}
$$

This is impossible. If $m \equiv 3,7(\bmod 8)$, since $\left(\begin{array}{c}m+2 n+k-3 \\ 2\end{array}\right)+\left(\begin{array}{l}k \\ 2\end{array}\right)=1$, taking $\left(\sigma_{1}(x)+\left(\begin{array}{l}k \\ 1\end{array}\right)\right)^{3}\left(\sigma_{2}(x)+\left(\begin{array}{c}k \\ 2\end{array}\right)+\left(\begin{array}{c}k-1 \\ 1\end{array}\right)\left(\sigma_{1}(x)+\left(\begin{array}{l}k \\ 1\end{array}\right)\right)\right)+\left(\sigma_{1}(x)+\left(\begin{array}{l}k \\ 1\end{array}\right)\right)^{4}+\varepsilon\left(\sigma_{1}(x)+\left(\begin{array}{l}k \\ 1\end{array}\right)\right)^{5}$, we have

$$
\begin{aligned}
0 & =\frac{a^{3}\left(1+\sigma_{2}(y)\right)+a^{4}+\varepsilon a^{5}}{\omega v_{1}}[R P(3)]+\frac{c^{3}\left(c+\varepsilon c^{2}\right)+c^{4}+\varepsilon c^{5}}{1+\left(\begin{array}{c}
m+n+1 \\
2
\end{array}\right) c^{2}+\varepsilon c^{2}}[P(m, n)] \\
& =1+0 \\
& =1 .
\end{aligned}
$$

This contrary equation shows that $m \equiv 3,7(\bmod 8)$ is impossible.

Combining the above discussions, the Lemma 4.3 thus holds.

LEMMA 4.4. There does not exist the involution $\left(T, M^{m+2 n+k}\right)$ for which $n$ is even and $m \equiv 1(\bmod 4)$.

Proof. Suppose that $\left(T, M^{m+2 n+k}\right)$ exists when $n$ is even and $m \equiv 1$ $(\bmod 4)$. By direct computations, we have

$$
\sigma_{1}(1+y, z)\left(v_{1} \rightarrow R P(3)\right)=\left(\begin{array}{c}
m+2 n+k-3 \\
1
\end{array}\right)+a
$$

and

$$
\sigma_{1}(1+y, z)\left(v_{2} \rightarrow P(m, n)\right)=\left(\begin{array}{c}
k \\
1
\end{array}\right)+\sigma_{1}(y) .
$$

Taking $\left(\sigma_{1}(x)+\left(\begin{array}{l}k \\ 1\end{array}\right)\right)^{3}$ and using Theorem 3.1, we have

$$
0=\frac{a^{3}}{\omega v_{1}}[R P(3)]+\frac{\left(\sigma_{1}(y)\right)^{3}}{\omega v_{2}}[P(m, n)]=1+\frac{\left(\sigma_{1}(y)\right)^{3}}{\omega v_{2}}[P(m, n)]
$$

and thus $\left(\sigma_{1}(y)\right)^{3} /\left(\omega v_{2}\right)[P(m, n)]=1$. Furthermore, it follows that $\omega_{1} v_{2}=c$ and $m$ is more than or equal to 5 . Again using [5; p. 317, Lemma], we have

$$
\sigma_{2}(1+y, z)\left(v_{1} \rightarrow R P(3)\right)=\left(\begin{array}{c}
k-2 \\
2
\end{array}\right)+\left(\begin{array}{c}
k+1 \\
1
\end{array}\right) a+\sigma_{2}(y)
$$


and

$$
\sigma_{2}(1+y, z)\left(v_{2} \rightarrow P(m, n)\right)=\left(\begin{array}{l}
k \\
2
\end{array}\right)+\left(\begin{array}{c}
k-1 \\
1
\end{array}\right) c+\sigma_{2}(y)+\sigma_{2}(z) .
$$

We proceed as follows:

(i) If $\sigma_{2}(y)+\sigma_{2}(z)=0$ in (4.2), taking

$$
\left(\sigma_{1}(x)+\left(\begin{array}{l}
k \\
1
\end{array}\right)\right)^{3}\left(\sigma_{2}(x)+\left(\begin{array}{l}
k \\
2
\end{array}\right)+\left(\begin{array}{c}
k+1 \\
1
\end{array}\right)\left(\sigma_{1}(x)+\left(\begin{array}{l}
k \\
1
\end{array}\right)\right)\right)
$$

we can obtain the following contrary equation

$$
0=\frac{a^{3}\left(1+\sigma_{2}(y)\right)}{\omega v_{1}}[R P(3)]+\frac{c^{3} \times 0}{\omega v_{2}}[P(m, n)]=1+0=1 .
$$

(ii) If $\sigma_{2}(y)+\sigma_{2}(z)=\varepsilon c^{2}+d$ (here $\left.\varepsilon \in Z_{2}\right)$ in (4.2), choose

$$
\left(\sigma_{1}(x)+\left(\begin{array}{l}
k \\
1
\end{array}\right)\right)^{m}\left(\sigma_{2}(x)+\left(\begin{array}{l}
k \\
2
\end{array}\right)+\left(\begin{array}{c}
k+1 \\
1
\end{array}\right)\left(\sigma_{1}(x)+\left(\begin{array}{l}
k \\
1
\end{array}\right)\right)\right)^{n}
$$

of degree less than $m+2 n+k$, we have

$$
0=\frac{a^{m}\left(1+\sigma_{2}(y)\right)^{n}}{\omega v_{1}}[R P(3)]+\frac{c^{m}\left(\varepsilon c^{2}+d\right)^{n}}{\omega v_{2}}[P(m, n)]=0+1=1 .
$$

This leads to a contradiction.

(iii) If $\sigma_{2}(y)+\sigma_{2}(z)=c^{2}$ in (4.2), choose

$$
\begin{aligned}
& \left(\sigma_{1}(x)+\left(\begin{array}{c}
k \\
1
\end{array}\right)\right)^{3} \\
& \quad \times\left(\sigma_{2}(x)+\left(\begin{array}{l}
k \\
2
\end{array}\right)+\left(\begin{array}{c}
k+1 \\
1
\end{array}\right)\left(\sigma_{1}(x)+\left(\begin{array}{l}
k \\
1
\end{array}\right)\right)\right)+\left(\sigma_{1}(x)+\left(\begin{array}{l}
k \\
1
\end{array}\right)\right)^{5},
\end{aligned}
$$

we have

$$
\begin{aligned}
0 & =\frac{a^{3}\left(1+\sigma_{2}(y)\right)+a^{5}}{\omega v_{1}}[R P(3)]+\frac{c^{3} c^{2}+c^{5}}{\omega v_{2}}[P(m, n)] \\
& =1+0 \\
& =1
\end{aligned}
$$

but this is impossible.

Combining the above discussions, we complete the proof of the Lemma 4.4.

Recall that for even $n$ and $m \equiv 3(\bmod 4), \omega v_{2}=(1+c)^{s}(1+c+d)^{t}$. Let $2^{N}>\max \{m, n\}, 2^{M}>m$. We have 
Lemma 4.5. Suppose that $\left(T, M^{m+2 n+k}\right)$ exists for which $n$ is even, $m \equiv 3$ $(\bmod 4)$ and $\omega v_{1}=1+a$. Then

$$
E(n) \subset E\left(2^{N+1}-t\right) \text { and } E(m) \subset E\left(2^{M+1}+2^{N+1}-s-t-n\right) .
$$

Proof. Choose symmetric polynomial function 1, we have

$$
\begin{aligned}
0 & =\frac{1}{1+a}[R P(3)]+\frac{1}{(1+c)^{s}(1+c+d)^{t}}[P(m, n)] \\
& =1+(1+c)^{2^{M+1}-s}(1+c+d)^{2^{N+1}-t}[P(m, n)] \\
& =1+\left(\begin{array}{c}
2^{N+1}-t \\
n
\end{array}\right)\left(\begin{array}{c}
2^{M+1}+2^{N+1}-s-t-n \\
m
\end{array}\right),
\end{aligned}
$$

and thus the result holds by Lucas Theorem.

In virtue of $\omega v_{2}=(1+c)^{s}(1+c+d)^{t}$ with even $n$ and $m \equiv 3(\bmod 4)$, we may assume that $E(s) \subset\left\{i \mid 2^{l} \leq m\right\}, E(t) \subset\left\{j \mid 2^{j} \leq n\right\}$ because $c^{x}=0, d^{y}=0$ for $x>m, y>n$, and $(1+c)^{2^{i}}=1,(1+d)^{2^{\jmath}}=1$ for $2^{l}>m, 2^{\jmath}>n$.

Lemma 4.6. Let $n$ is even and $t$ is odd. If $E(n) \subset E\left(2^{N+1}-t\right)$, then $t<n$.

Proof. First, we prove that $E(n) \cap E(t)=\Phi$ (empty set). Since $E\left(2^{N+1}-1\right)=\{0,1, \ldots, N\}$, it follows that each element in $E(t-1)$ doesn't belong to $E\left(2^{N+1}-t\right)$. Hence $E(n) \cap E(t-1)=\Phi$. Since $t$ is odd and $n$ is even, we have $E(t)=E(t-1) \cup\{0\}$ and $0 \notin E(n)$. Therefore we can obtain $E(n) \cap E(t)=\Phi$. Next, let $u=\max \{\alpha \mid \alpha \in E(t)\}, \quad v=\max \{\beta \mid \beta \in E(n)\}$. It is obvious that $u \neq v$ since $E(n) \cap E(t)=\Phi$. Because $E(t) \subset\left\{j \mid 2^{J} \leq n\right\}$, we have $u<v$ and thus $E(t) \subset E\left(2^{v}-1\right)$. Furthermore, we have $n \geq 2^{v}>t$. This completes the proof.

LEMMA 4.7. If $n$ is even, $n \equiv 3(\bmod 4)$ and $\omega v_{1}=1+a$, then $\left(T, M^{m+2 n+k}\right)$ doesn't exist except for the following cases: either

or

(I) $\omega v_{2}=(1+c)^{2}(1+c+d), m=3, n=4 l+2,4 \leq k \leq 6 ;$

(II) $\omega v_{2}=1+c+d, m=3, n=4 l, 2 \leq k \leq 4 A-3$

where $A=\min \{\alpha \mid \alpha>0$ and $E(\alpha) \subset E(2 L)\}$.

Proof. Suppose that $\left(T, M^{m+2 n+k}\right)$ exists under the condition that $n$ is even, $m \equiv 3(\bmod 4)$ and $\omega v_{1}=1+a$. By direct computations, we have

and

$$
\sigma_{1}(1+y, z)\left(v_{1} \rightarrow R P(3)\right)=\left(\begin{array}{c}
m+2 n+k-3 \\
1
\end{array}\right)+a
$$

$$
\sigma_{1}(1+y, z)\left(v_{2} \rightarrow P(m, n)\right)=\left(\begin{array}{c}
k \\
1
\end{array}\right)+c .
$$


Taking $\left(\sigma_{1}(x)+\left(\begin{array}{l}k \\ 1\end{array}\right)\right)^{m}$, then

$$
\begin{aligned}
0 & =\frac{a^{m}}{1+a}[R P(3)]+\frac{c^{m}}{(1+c)^{s}(1+c+d)^{t}}[P(m, n)] \\
& =\frac{a^{m}}{1+a}[R P(3)]+\left(\begin{array}{c}
2^{N+1}-t \\
n
\end{array}\right) .
\end{aligned}
$$

By Lemma 4.5, we have $a^{m} /(1+a)[R P(3)]=1$, and thus $m$ must be 3 . Since

$$
\sigma_{2}(1+y, z)\left(v_{1} \rightarrow R P(3)\right)=\left(\begin{array}{c}
2 n+k \\
2
\end{array}\right)+\left(\begin{array}{c}
2 n+k-1 \\
1
\end{array}\right) a
$$

and

$$
\begin{aligned}
\sigma_{2}(1+y, z)\left(v_{2} \rightarrow P(3, n)\right)= & \left(\begin{array}{l}
k \\
2
\end{array}\right)+\left(\begin{array}{c}
k-1 \\
1
\end{array}\right) c+\left(\begin{array}{c}
n+4 \\
2
\end{array}\right) c^{2} \\
& +\left(\begin{array}{c}
s+t \\
2
\end{array}\right) c^{2}+\left(\begin{array}{l}
t \\
1
\end{array}\right) d+d,
\end{aligned}
$$

take $\left(\sigma_{1}(x)+\left(\begin{array}{l}k \\ 1\end{array}\right)\right)^{3}\left(\sigma_{2}(x)+\left(\begin{array}{l}k \\ 2\end{array}\right)\right)^{n}$, we may conclude that

$$
\begin{aligned}
0 & =\frac{0}{1+a}[R P(3)]+\frac{c^{3}\left(\left(\begin{array}{l}
t \\
1
\end{array}\right) d+d\right)^{n}}{(1+c)^{s}(1+c+d)^{t}}[P(m, n)] \\
& =0+\left(\begin{array}{l}
t \\
1
\end{array}\right)+1 .
\end{aligned}
$$

and thus $\left(\begin{array}{l}t \\ 1\end{array}\right)=0$ is impossible. Let $\left(\begin{array}{l}t \\ 1\end{array}\right)=1$, i.e., $t$ is odd, by Lemma 4.5 , we know that $s+t$ is odd, and thus $s$ must be even. Furthermore, we can obtain $s=0$ or 2 since $E(s) \subset\left\{i \mid 2^{l} \leq m\right\}$ and $m=3$. On the other hand, since $t$ is odd and $n$ is even, by Lemma 4.6, we have $t<n$. Therefore, it follows that the highest degree term in $\omega v_{2}=(1+c)^{s}(1+c+d)^{t}$ is $c^{s} d^{t} \neq 0$, and thus $k \geq$ $s+2 t$. We proceed as follows:

$$
\text { If } \begin{aligned}
& \left(\begin{array}{c}
n+4 \\
2
\end{array}\right)+\left(\begin{array}{c}
s+t \\
2
\end{array}\right)=1 \text {, choose } \\
& \qquad\left(\sigma_{1}(x)+\left(\begin{array}{l}
k \\
1
\end{array}\right)\right)\left(\sigma_{2}(x)+\left(\begin{array}{l}
k \\
2
\end{array}\right)+\left(\begin{array}{c}
k-1 \\
1
\end{array}\right)\left(\sigma_{1}(x)+\left(\begin{array}{c}
k \\
1
\end{array}\right)\right)\right),
\end{aligned}
$$

by Theorem 3.1 , we may obtain a contradiction.

If $\left(\begin{array}{c}n+4 \\ 2\end{array}\right)+\left(\begin{array}{c}s+t \\ 2\end{array}\right)=0$, we have

$$
\sigma_{4}(1+y, z)\left(v_{1} \rightarrow R P(3)\right)=\left(\begin{array}{c}
2 n+k \\
4
\end{array}\right)+\left(\begin{array}{c}
2 n+k-1 \\
3
\end{array}\right) a
$$

and 


$$
\begin{aligned}
& \sigma_{4}(1+y, z)\left(v_{2} \rightarrow P(3, n)\right) \\
& =\left\{\begin{array}{l}
\left(\begin{array}{l}
k \\
4
\end{array}\right)+\left(\begin{array}{c}
k-1 \\
3
\end{array}\right) c+d+c d+c^{2}+\left(\begin{array}{l}
t \\
2
\end{array}\right) d^{2}, \quad \text { if }\left(\begin{array}{c}
n+4 \\
2
\end{array}\right)=\left(\begin{array}{c}
s+t \\
2
\end{array}\right)=1 \\
\left(\begin{array}{l}
k \\
4
\end{array}\right)+\left(\begin{array}{c}
k-1 \\
3
\end{array}\right) c+d+c d+d^{2}+\left(\begin{array}{l}
t \\
2
\end{array}\right) d^{2}, \quad \text { if }\left(\begin{array}{c}
n+4 \\
2
\end{array}\right)=\left(\begin{array}{c}
s+t \\
2
\end{array}\right)=0 .
\end{array}\right.
\end{aligned}
$$

Consider the following

(i) When $\left(\begin{array}{c}n+4 \\ 2\end{array}\right)=\left(\begin{array}{c}s+t \\ 2\end{array}\right)=1$, i.e., $n=4 l+2$, since $E(n) \cap E(t)=\Phi$ (see the proof of Lemma 4.6), it follows that $1 \notin E(t)$, i.e., $\left(\begin{array}{l}t \\ 2\end{array}\right)=0$. Furthermore, from $\left(\begin{array}{c}s+t \\ 2\end{array}\right)=1$ we have $s=2$. If $t>1$, choose

$$
\left(\sigma_{1}(x)+\left(\begin{array}{l}
k \\
1
\end{array}\right)\right)^{3}\left(\sigma_{4}(x)+\left(\begin{array}{c}
2 n+k \\
4
\end{array}\right)\right)^{t-1}
$$

by Theorem 3.1 we have

$$
\begin{aligned}
0 & =\frac{0}{1+a}[R P(3)]+\frac{c^{3}(1+d)^{t-1}}{(1+c)^{2}(1+c+d)^{t}}[P(3,4 l+2)] \\
& =0+\frac{c^{3}}{1+d}[P(3,4 l+2)] \\
& =1 .
\end{aligned}
$$

This shows that for $t>1$, the involution $\left(T, M^{7+8 l+k}\right)$ doesn't exist. Hence, in the next discussions, it suffices to check the following case:

$$
s=2, \quad t=1, \quad m=3, \quad n=4 l+2, \quad k \geq 4 .
$$

By direct computations, we have

$$
\sigma_{8 l+6}(1+y, z)\left(v_{1} \rightarrow R P(3)\right)=\left(\begin{array}{c}
8 l+4+k \\
8 l+6
\end{array}\right)+\left(\begin{array}{c}
8 l+3+k \\
8 l+5
\end{array}\right) a
$$

and

$$
\begin{aligned}
\sigma_{8 l+6}(1+y, z)\left(v_{2} \rightarrow P(3,4 l+2)\right) \\
=d^{4 l+2}+c d^{4 l+2}+c^{3} c^{4 l+1}+\left(\left(\begin{array}{c}
k-1 \\
2
\end{array}\right)+\left(\begin{array}{c}
k-4 \\
2
\end{array}\right)\right) c^{2} d^{4 l+1} \\
\quad+\text { terms of degree less than } 8 l+4 .
\end{aligned}
$$

If $k>6$, choose 
INVOLUTIONS FIXING THE DISJOINT UNION OF 3-REAL PROJECTIVE SPACE 201

$$
\begin{aligned}
f(x)= & \sigma_{8 l+6}(x)\left(\sigma_{4}(x)+\left(\begin{array}{c}
8 l+4+k \\
4
\end{array}\right)+\left(\begin{array}{c}
k-1 \\
3
\end{array}\right)\left(\sigma_{1}(x)+\left(\begin{array}{l}
k \\
1
\end{array}\right)\right)\right) \\
& \times\left(\sigma_{1}(x)+\left(\begin{array}{l}
k \\
1
\end{array}\right)\right)^{3}
\end{aligned}
$$

with $\operatorname{deg} f=8 l+13<m+2 n+k$, since

$$
f(1+y, z)\left(v_{1} \rightarrow R P(3)\right)=\left(\left(\begin{array}{c}
8 l+4+k \\
8 l+6
\end{array}\right)+\left(\begin{array}{c}
8 l+3+k \\
8 l+5
\end{array}\right) a\right) \times 0 \times a^{3}=0
$$

and

$$
\begin{aligned}
f(1+y, z)\left(v_{2} \rightarrow P(3,4 l+2)\right) & \\
& =\left(d^{4 l+2}+\text { terms of degree less than } 8 l+4\right)(1+d) c^{3},
\end{aligned}
$$

by Theorem 3.1, then

$$
\begin{aligned}
0= & \frac{0}{1+a}[R P(3)] \\
& +\frac{c^{3}(1+d)\left(d^{4 l+2}+\text { terms of degree less than } 8 l+4\right)}{(1+c)^{2}(1+c+d)}[P(3,4 l+2)] \\
= & 0+\left(c^{3} d^{4 l+2}+\text { terms of degree less than } 8 l+7\right)[P(3,4 l+2)] \\
= & 1,
\end{aligned}
$$

but this is a contradiction.

(ii) When $\left(\begin{array}{c}n+4 \\ 2\end{array}\right)=\left(\begin{array}{c}s+t \\ 2\end{array}\right)=0$, i.e., $n=4 l$, taking

$$
\left(\sigma_{1}(x)+\left(\begin{array}{l}
k \\
1
\end{array}\right)\right)^{3}\left(\sigma_{4}(x)+\left(\begin{array}{l}
k \\
4
\end{array}\right)+1\right)^{t}
$$

by Theorem 3.1 , we may obtain that $\left(\begin{array}{l}t \\ 2\end{array}\right)=1$ is impossible. Let $\left(\begin{array}{l}t \\ 2\end{array}\right)=0$, since $\left(\begin{array}{c}s+t \\ 2\end{array}\right)=0$, then $s=0$. Taking

$$
\left(\sigma_{1}(x)+\left(\begin{array}{l}
k \\
1
\end{array}\right)\right)^{3}\left(\sigma_{4}(x)+\left(\begin{array}{l}
k \\
4
\end{array}\right)\right)^{t-1}
$$

by Theorem 3.1, we may easily show that $t>1$ is impossible. Now we need only to check the following case:

$$
s=0, \quad t=1, \quad m=3, \quad n=4 l, \quad k \geq 2 .
$$

Let $A=\min \{\alpha \mid \alpha>0$ and $E(\alpha) \subset E(2 l)\}$. If $k+3>4 A$, taking

$$
\left(\sigma_{4}(x)+\left(\begin{array}{l}
k \\
4
\end{array}\right)+\left(\begin{array}{c}
k-1 \\
3
\end{array}\right)\left(\sigma_{1}(x)+\left(\begin{array}{l}
k \\
1
\end{array}\right)\right)\right)^{2 l+A}
$$


and using Theorem 3.1, then

$$
\begin{aligned}
0 & =\frac{0}{1+a}[R P(3)]+\frac{\left(d+c d+d^{2}\right)^{2 l+A}}{1+c+d}[P(3,4 l)] \\
& =0+d^{2 l+A}(1+c+d)^{2 l+A-1}[P(3,4 l)] \\
& =d^{2 l+A}\left(\left(\begin{array}{c}
2 l+A-1 \\
2 l-A
\end{array}\right) d^{2 l-A}(1+c)^{2 A-1}\right)[P(3,4 l)] \\
& =\left(\begin{array}{c}
2 l+A-1 \\
2 A-1
\end{array}\right)\left(\begin{array}{c}
2 A-1 \\
3
\end{array}\right)
\end{aligned}
$$

From the definition of $A$, it is easy to see that $A=2^{i}$ for some $i$. Therefore we may conclude that $E(3)=\{0,1\} \subset E(2 A-1)$ and

$$
E(2 A-1)=E(A) \cup E(A-1) \subset E(2 l) \cup E(A-1)=E(2 l+A-1) .
$$

Furthermore, by Lucas Theorem, we have

$$
\left(\begin{array}{c}
2 l+A-1 \\
2 A-1
\end{array}\right)\left(\begin{array}{c}
2 A-1 \\
3
\end{array}\right)=1 .
$$

But this leads to a contradiction.

Combining the above, consequently we complete the proof.

Now consider the case in which $n$ is even, $m \equiv 3(\bmod 4)$ and $\omega v_{1}=$ $(1+a)^{3}$.

LeMma 4.8. Suppose $\left(T, M^{m+2 n+k}\right)$ exists under the condition that $n$ is even, $m \equiv 3(\bmod 4)$ and $\omega v_{1}=(1+a)^{3}$. Then

$$
E(n) \subset E\left(2^{N+1}-t\right)
$$

and $s+t$ is odd.

Proof. Choose symmetric polynomial function $\left(\sigma_{1}(x)+\left(\begin{array}{l}k \\ 1\end{array}\right)\right)^{2}$, we have

$$
\begin{aligned}
0 & =\frac{a^{2}}{(1+a)^{3}}[R P(3)]+\frac{\left(\sigma_{1}(y)\right)^{2}}{(1+c)^{s}(1+c+d)^{t}}[P(m, n)] \\
& =1+\frac{\left(\sigma_{1}(y)\right)^{2}}{(1+c)^{s}(1+c+d)^{t}}[P(m, n)]
\end{aligned}
$$

and thus $\left(\sigma_{1}(y)\right)^{2} /\left((1+c)^{s}(1+c+d)^{t}\right)[P(m, n)]=1$. This means that $\omega_{1} v_{2} \neq 0$. Let $\omega_{1} v_{2}=c$. Then we have 


$$
\begin{aligned}
1 & =\frac{\left(\sigma_{1}(y)\right)^{2}}{(1+c)^{S}(1+c+d)^{t}}[P(m, n)] \\
& =\frac{c^{2}}{(1+c)^{S}(1+c+d)^{t}}[P(m, n)] \\
& =c^{2}(1+c)^{2^{M+1}-s}(1+c+d)^{2^{N+1}-t}[P(m, n)] \\
& =\left(\begin{array}{c}
2^{N+1}-t \\
n
\end{array}\right)\left(\begin{array}{c}
2^{M+1}+2^{N+1}-s-t-n \\
m-2
\end{array}\right),
\end{aligned}
$$

and thus the result holds by Lucas Theorem.

Note that for the case in which $n$ is even, $m \equiv 3(\bmod 4)$ and $\omega v_{1}=(1+a)^{3}$, it is easy to see that Lemma 4.6 still holds.

LEMMA 4.9. If $n$ is even, $m \equiv 3(\bmod 4)$ and $\omega v_{1}=(1+a)^{3}$, then $\left(T, M^{m+2 n+k}\right)$ doesn't exist except for the following cases: either

(I) $\omega v_{2}=1+c+d, m=3, n=4 l+2,2 \leq k \leq 4$;

or

(II) $\omega v_{2}=(1+c)^{2}(1+c+d), \quad m=3, n=4 l, 4 \leq k \leq 4 A-1$ where $A=$ $\min \{\alpha \mid \underline{\alpha}>0$ and $E(\alpha) \subset E(2 l)\}$.

Proof. Suppose $\left(T, M^{m+2 n+k}\right)$ exists when $n$ is even, $m \equiv 3(\bmod 4)$ and $\omega v_{1}=(1+a)^{3}$. Choose $\left(\sigma_{1}(x)+\left(\begin{array}{l}k \\ 1\end{array}\right)\right)^{m}$, it follows that

$$
\begin{aligned}
0 & =\frac{a^{m}}{(1+a)^{3}}[R P(3)]+\frac{c^{m}}{(1+c)^{s}(1+c+d)^{t}}[P(m, n)] \\
& =\frac{a^{m}}{(1+a)^{3}}[R P(3)]+\left(\begin{array}{c}
2^{N+1}-t \\
n
\end{array}\right) \\
& =\frac{a^{m}}{(1+a)^{3}}[R P(3)]+1 \text { (by Lemma 4.8) }
\end{aligned}
$$

and thus $m=2$ or 3 . Furthermore, we have that $m=3$ since $m \equiv 3(\bmod 4)$. By direct computations, we have

and

$$
\sigma_{2}(1+y, z)\left(v_{1} \rightarrow R P(3)\right)=\left(\begin{array}{c}
2 n+k \\
2
\end{array}\right)+\left(\begin{array}{c}
2 n+k-1 \\
1
\end{array}\right) a+a^{2}
$$

$$
\begin{aligned}
\sigma_{2}(1+y, z)\left(v_{2} \rightarrow P(3, n)\right)= & \left(\begin{array}{l}
k \\
2
\end{array}\right)+\left(\begin{array}{c}
k-1 \\
1
\end{array}\right) c \\
& +\left(\begin{array}{c}
n+4 \\
2
\end{array}\right) c^{2}+\left(\begin{array}{c}
s+t \\
2
\end{array}\right) c^{2}+\left(\begin{array}{l}
t \\
1
\end{array}\right) d+d
\end{aligned}
$$


As in the proof of Lemma 4.7, choose $\left(\sigma_{1}(x)+\left(\begin{array}{l}k \\ 1\end{array}\right)\right)^{3}\left(\sigma_{2}(x)+\left(\begin{array}{l}k \\ 2\end{array}\right)\right)^{n}$, we may obtain that $\left(\begin{array}{l}t \\ 1\end{array}\right)=0$ is impossible. So $\left(\begin{array}{l}t \\ 1\end{array}\right)=1$. Furthermore, by Lemma 4.8 we have that $s$ must be even, and thus $s=0$ or 2. Again by Lemma 4.8 and Lemma 4.6, it follows that $t<n$. Hence $k \geq s+2 t$. We proceed as follows:

If $\left(\begin{array}{c}n+4 \\ 2\end{array}\right)+\left(\begin{array}{c}s+t \\ 2\end{array}\right)=0$, take

$$
\left(\sigma_{1}(x)+\left(\begin{array}{l}
k \\
1
\end{array}\right)\right)\left(\sigma_{2}(x)+\left(\begin{array}{l}
k \\
2
\end{array}\right)+\left(\begin{array}{c}
k-1 \\
1
\end{array}\right)\left(\sigma_{1}(x)+\left(\begin{array}{l}
k \\
1
\end{array}\right)\right),\right.
$$

we may conclude that this case is impossible.

If $\left(\begin{array}{c}n+4 \\ 2\end{array}\right)+\left(\begin{array}{c}s+t \\ 2\end{array}\right)=1$, consider

$$
\begin{aligned}
\sigma_{4}(1+y, z)\left(v_{1} \rightarrow R P(3)\right)= & \left(\begin{array}{c}
2 n+k \\
4
\end{array}\right)+\left(\begin{array}{c}
2 n+k-1 \\
3
\end{array}\right) a \\
& +\left(\begin{array}{c}
2 n+k-2 \\
2
\end{array}\right) a^{2}+\left(\begin{array}{c}
2 n+k-3 \\
1
\end{array}\right) a^{3}
\end{aligned}
$$

and

$$
\begin{aligned}
& \sigma_{4}(1+y, z)\left(v_{2} \rightarrow P(3, n)\right) \\
& =\left\{\begin{array}{r}
\left(\begin{array}{l}
k \\
4
\end{array}\right)+\left(\begin{array}{c}
k-1 \\
3
\end{array}\right) c+\left(\begin{array}{l}
k \\
2
\end{array}\right) c^{2}+d+c d+\left(\begin{array}{c}
k-1 \\
1
\end{array}\right) c^{3}+\left(\begin{array}{l}
t \\
2
\end{array}\right) d^{2}, \\
\text { if }\left(\begin{array}{c}
n+4 \\
2
\end{array}\right)=1,\left(\begin{array}{c}
s+t \\
2
\end{array}\right)=0 \\
\left(\begin{array}{l}
k \\
4
\end{array}\right)+\left(\begin{array}{c}
k-1 \\
3
\end{array}\right) c+\left(\begin{array}{c}
k-2 \\
2
\end{array}\right) c^{2}+d+c d+\left(\begin{array}{c}
k-3 \\
1
\end{array}\right) c^{3}+d^{2}+\left(\begin{array}{c}
t \\
2
\end{array}\right) d^{2}, \\
\text { if }\left(\begin{array}{c}
n+4 \\
2
\end{array}\right)=0,\left(\begin{array}{c}
s+t \\
2
\end{array}\right)=1 .
\end{array}\right.
\end{aligned}
$$

Now consider the following cases:

When $\left(\begin{array}{c}n+4 \\ 2\end{array}\right)=1$ (i.e. $\left.n=4 l+2\right)$ and $\left(\begin{array}{c}s+t \\ 2\end{array}\right)=0$, by the proof of Lemma 4.6 we have $E(n) \cap E(t)=\Phi$. Thus $1 \notin E(t)$, i.e. $\left(\begin{array}{l}t \\ 2\end{array}\right)=0$. Furthermore, since $\left(\begin{array}{c}s+t \\ 2\end{array}\right)$ $=0$, if follows that $s=0$. If $t>1$, take

$$
\left(\sigma_{1}(x)+\left(\begin{array}{c}
k \\
1
\end{array}\right)\right)^{3}\left(\sigma_{4}(x)+\left(\begin{array}{c}
2 n+k \\
4
\end{array}\right)\right)^{t-1},
$$

it is easy to see that $t>1$ is impossible. Moreover, it needs to check the following case:

$$
s=0, \quad t=1, \quad m=3, \quad n=4 l+2, \quad k \geq 2 .
$$


INVOLUTIONS FIXING THE DISJOINT UNION OF 3-REAL PROJECTIVE SPACE 205 By computations, we have

and

$$
\begin{aligned}
\sigma_{8 l+6}(1+y, z)\left(v_{1} \rightarrow R P(3)\right)= & \left(\begin{array}{c}
8 l+4+k \\
8 l+6
\end{array}\right)+\left(\begin{array}{c}
8 l+3+k \\
8 l+5
\end{array}\right) a \\
& +\left(\begin{array}{c}
8 l+2+k \\
8 l+4
\end{array}\right) a^{2}+\left(\begin{array}{c}
8 l+1+k \\
8 l+3
\end{array}\right) a^{3}
\end{aligned}
$$

$$
\begin{aligned}
\sigma_{8 l+6}(1+y, z)\left(v_{2} \rightarrow P(3,4 l+2)\right) \\
=d^{4 l+2}+c d^{4 l+2}+\left(\left(\begin{array}{c}
k-1 \\
2
\end{array}\right)+\left(\begin{array}{c}
k-2 \\
2
\end{array}\right)\right) c^{2} d^{4 l+1} \\
\quad+\text { terms of degree less than } 8 l+4 .
\end{aligned}
$$

If $k>4$, take $\sigma_{8 l+6}(x)\left(\sigma_{4}(x)+\left(\begin{array}{c}8 l+4+k \\ 4\end{array}\right)+\left(\begin{array}{c}k-1 \\ 3\end{array}\right)\left(\sigma_{1}(x)+\left(\begin{array}{l}k \\ 1\end{array}\right)\right)+\left(\begin{array}{c}k-2 \\ 2\end{array}\right)\left(\sigma_{1}(x)+\left(\begin{array}{l}k \\ 1\end{array}\right)\right)^{2}\right.$ $\left.+\left(\begin{array}{c}k-3 \\ 1\end{array}\right)\left(\sigma_{1}(x)+\left(\begin{array}{l}k \\ 1\end{array}\right)\right)^{3}\right)\left(\sigma_{1}(x)+\left(\begin{array}{l}k \\ 1\end{array}\right)\right)$ of degree $8 l+11$ less than $m+2 n+k$, by Theorem 3.1, then

$$
\begin{aligned}
0= & \frac{0}{1+a}[R P(3)] \\
& +\frac{c\left(1+c^{2}+d+c d\right)\left(d^{4 l+2}+c d^{4 l+2}+\left(\left(\begin{array}{c}
k-1 \\
2
\end{array}\right)+\left(\begin{array}{c}
k-2 \\
2
\end{array}\right)\right) c^{2} d^{4 l+1}\right)}{1+c+d} \\
& \times[P(3,4 l+2)] \\
& +\frac{c\left(1+c^{2}+d+c d\right)(\text { terms of degree less than } 8 l+4)}{1+c+d} \\
& \quad \times[P(3,4 l+2)] \\
= & \frac{c(1+c)(1+c+d)\left(d^{4 l+2}+c d^{4 l+2}+\left(\left(\begin{array}{c}
k-1 \\
2
\end{array}\right)+\left(\begin{array}{c}
k-2 \\
2
\end{array}\right)\right) c^{2} d^{4 l+1}\right)}{1+c+d} \\
& \times[P(3,4 l+2)] \\
& +\frac{c(1+c)(1+c+d)(\text { terms of degree less than } 8 l+4)}{1+c+d}[P(3,4 l+2)] \\
& +c(1+c)\left(d^{4 l+2}+c d^{4 l+2}+\left(\left(\begin{array}{c}
k-1 \\
2
\end{array}\right)+\left(\begin{array}{c}
k-2 \\
2
\end{array}\right)\right) c^{2} d^{4 l+1}\right)[P(3,4 l+2)] \\
= & 1,
\end{aligned}
$$

but this is a contradiction. 
When $\left(\begin{array}{c}n+4 \\ 2\end{array}\right)=0$ (i.e. $\left.n=4 l\right)$ and $\left(\begin{array}{c}s+t \\ 2\end{array}\right)=1$, take

$$
\left(\sigma_{1}(x)+\left(\begin{array}{l}
k \\
1
\end{array}\right)\right)^{3}\left(\sigma_{4}(x)+\left(\begin{array}{l}
k \\
4
\end{array}\right)+1\right)^{t},
$$

it follows that $\left(\begin{array}{l}t \\ 2\end{array}\right)=1$ is impossible. Let $\left(\begin{array}{l}t \\ 2\end{array}\right)=0$. Since $\left(\begin{array}{c}s+t \\ 2\end{array}\right)=1$, we have $s=2$. If $t>1$, choose

$$
\left(\sigma_{1}(x)+\left(\begin{array}{l}
k \\
1
\end{array}\right)\right)^{3}\left(\sigma_{4}(x)+\left(\begin{array}{l}
k \\
4
\end{array}\right)\right)^{t-1},
$$

then we conclude that this case is impossible. Furthermore, it needs merely to check the following case:

$$
s=2, \quad t=1, \quad m=3, \quad n=4 l, \quad k \geq 4 .
$$

If $\quad k+1>4 A$, choose $\left(\sigma_{1}(x)+\left(\begin{array}{l}k \\ 1\end{array}\right)+1\right)^{2}\left(\sigma_{4}(x)+\left(\begin{array}{c}k \\ 4\end{array}\right)+\left(\begin{array}{c}k-1 \\ 3\end{array}\right)\left(\sigma_{1}(x)+\left(\begin{array}{l}k \\ 1\end{array}\right)\right)+\right.$ $\left.\left(\begin{array}{c}k-2 \\ 2\end{array}\right)\left(\sigma_{1}(x)+\left(\begin{array}{l}k \\ 1\end{array}\right)\right)^{2}+\left(\begin{array}{c}k-3 \\ 1\end{array}\right)\left(\sigma_{1}(x)+\left(\begin{array}{l}k \\ 1\end{array}\right)\right)^{3}\right)^{2 l+A}$, then we have

$$
\begin{aligned}
0 & =\frac{0}{1+a}[R P(3)]+\frac{(1+c)^{2}\left(d+c d+d^{2}\right)^{2 l+A}}{(1+c)^{2}(1+c+d)}[P(3,4 l)] \\
& =0+d^{2 l+A}(1+c+d)^{2 l+A-1}[P(3,4 l)] \\
& =\left(\begin{array}{c}
2 l+A-1 \\
2 A-1
\end{array}\right)\left(\begin{array}{c}
2 A-1 \\
3
\end{array}\right) .
\end{aligned}
$$

We know from the proof of Lemma 4.7 that

$$
\left(\begin{array}{c}
2 l+A-1 \\
2 A-1
\end{array}\right)\left(\begin{array}{c}
2 A-1 \\
3
\end{array}\right)=1 .
$$

Therefore, it follows that $k+1>4 A$ is impossible.

Together with the above discussions, this completes the proof.

Combining Lemmas 4.1, 4.2, 4.3, 4.4, 4.7, 4.9, we have

Proposition 4.10. There doesn't exist the involution $\left(T, M^{m+2 n+k}\right)$ such that $v_{1} \rightarrow R P(3)$ doesn't bound except for the following four cases:

(I) $\omega v_{1}=1+a, \omega v_{2}=(1+c)^{2}(1+c+d), m=3, n=4 l+2,4 \leq k \leq 6$;

(II) $\omega v_{1}=1+a, \omega v_{2}=1+c+d, m=3, n=4 l, 2 \leq k \leq 4 A-3$;

(III) $\omega v_{1}=(1+a)^{3}, \omega v_{2}=1+c+d, m=3, n=4 l+2,2 \leq k \leq 4$;

(IV) $\omega v_{1}=(1+a)^{3}, \quad \omega v_{2}=(1+c)^{2}(1+c+d), \quad m=3, \quad n=4 l, \quad 4 \leq k \leq$ $4 A-1$ where $A=\min \{\alpha \mid \alpha>0$ and $E(\alpha) \subset E(2 l)\}$.

Remark 4.1. It should be point out that if $n$ is even and $m=3$, even though $v_{1} \rightarrow R P(3)$ bounds, we may easily prove that $\left(T, M^{m+2 n+k}\right)$ doesn't exist. 


\section{§5. The cases in which involutions exist}

In this section, we will prove that those involutions $\left(T, M^{m+2 n+k}\right)$ of the four cases stated in Proposition 4.10 must exist. Our main result is stated as follows:

THeOREM 5.1. Suppose that $\left(T, M^{m+2 n+k}\right)$ is an involution on a closed $(m+2 n+k)$-manifold with the fixed point set $F=R P(3) \sqcup P(m, n)(m, n>0)$, and with nonbounding normal bundle to $R P(3)$. Then there only exist those involutions $\left(T, M^{m+2 n+k}\right)$ satisfying the following four cases respectively:

(I) $\omega v_{1}=1+a, \omega v_{2}=(1+c)^{2}(1+c+d), m=3, n=4 l+2,4 \leq k \leq 6$;

(II) $\omega v_{1}=1+a, \omega v_{2}=1+c+d, m=3, n=4 l, 2 \leq k \leq 4 A-3$;

(III) $\omega v_{1}=(1+a)^{3}, \omega v_{2}=1+c+d, m=3, n=4 l+2,2 \leq k \leq 4$;

(IV) $\omega v_{1}=(1+a)^{3}, \omega v_{2}=(1+c)^{2}(1+c+d), m=3, n=4 l, 4 \leq k \leq 4 A-$ 1 where $A=\min \{\alpha \mid E(\alpha) \subset E(2 l)$ and $\alpha>0\}$.

For convenience, in the following proofs, let $\sigma_{i}(1+y, z)=\sigma_{l}, \sigma_{l}^{(1)}=$ $\sigma_{i}(1+y, z)\left(v_{1} \rightarrow R P(3)\right), \sigma_{l}^{(2)}=\sigma_{i}(1+y, z)\left(v_{2} \rightarrow P(m, n)\right)$, and $\sigma_{l}=0$ means $\sigma_{l}^{(1)}$ $=0, \sigma_{l}^{(2)}=0$.

As the sake of analogue of proof way, we will only prove the cases (I), (II) of Theorem 5.1. In fact, the proof method for the cases (I), (II) in Theorem 5.1 is not only the same as that for the cases (III), (IV), but also the involutions of the cases (I), (II) (i.e., case $\omega v_{1}=1+a$ ) possess completely the analogous structures as those of the cases (III), (IV), (i.e., cases $\left.\omega v_{1}=(1+a)^{3}\right)$. This is just seen from the results of Theorem 5.1 and the proofs of Lemmas 4.5, 4.6, 4.7, 4.8, and 4.9.

Recall that $\xi$ is a 1-plane bundle over $P(m, n)$ and $\eta$ is a 2-plane bundle over $P(m, n)$ (see $\S 2)$. Let $l$ be a canonical line bundle over $R P(3)$.

Proof of Theorem 5.1 (I). For $4 \leq k \leq 6$, since

$$
\imath \oplus(8 l+3+k) R \rightarrow R P(3) \sqcup 2 \xi \oplus \eta \oplus(k-4) R \rightarrow P(3,4 l+2)
$$

is bordant to $v_{1}^{8 l+4+k} \rightarrow R P(3) \sqcup v_{2}^{k} \rightarrow P(3,4 l+2)$ with $\omega v_{1}=1+a$ and $\omega v_{2}=$ $(1+c)^{2}(1+c+d)$, by Corollary 3.3 and Lemmas $3.4,3.5$ it suffices to show that the involution $\left(T, M^{8 l+12}\right)$ corresponding to the case $k=5$ of Theorem 5.1 (II) exists, and $M^{8 l+12}$ bounds. Moreover, by Theorem 3.1 and Proposition 3.2 it needs merely to prove that $v_{1}^{8 l+9} \rightarrow R P(3) \sqcup v_{2}^{5} \rightarrow P(3,4 l+2)$ with $\omega v_{1}=1+a$ and $\omega v_{2}=(1+c)^{2}(1+c+d)$ satisfies the following equation

$$
\frac{f(1+y, z)}{1+a}[R P(3)]+\frac{f(1+y, z)}{(1+c)^{2}(1+c+d)}[P(3,4 l+2)]=0
$$

for all symmetric polynomial $f(x)$ of degree less than or equal to $8 l+12$. For this, we first compute $\sigma_{l}$ for $0 \leq i \leq 8 l+12$. We proceed as follows. 
Using [5; p. 317, Lemma], it follows that

$$
\sigma_{l}^{(1)}=\left(\begin{array}{c}
8 l+9 \\
i
\end{array}\right)+\left(\begin{array}{c}
8 l+8 \\
i-1
\end{array}\right) a
$$

and

$$
\begin{aligned}
\sigma_{l}^{(2)}= & \sigma_{i}(z)+(1+c) \sigma_{i-1}(z)+\left(c^{2}+d\right) \sigma_{i-2}(z)+\left(c^{2}+d+c^{3}\right) \sigma_{l-3}(z) \\
& +\left(1+c^{2}+d+c^{2} d\right) \sigma_{l-4}(z)+(1+c)^{2}(1+c+d) \sigma_{i-5}(z) .
\end{aligned}
$$

From $\omega P(3,4 l+2)=(1+c)^{3}(1+c+d)^{4 l+3}$ we have that for $0 \leq j \leq l$,

$$
\begin{gathered}
\omega_{8 j} P(3,4 l+2)=\left(\begin{array}{c}
l \\
j
\end{array}\right) d^{4 j}+\left(\begin{array}{c}
l \\
j-1
\end{array}\right) c^{2} d^{4 j-1}, \omega_{8 j+1} P(3,4 l+2)=\left(\begin{array}{c}
l \\
j-1
\end{array}\right) c^{3} d^{4 j-1}, \\
\omega_{8 j+2} P(3,4 l+2)=\left(\begin{array}{c}
l \\
j
\end{array}\right)\left(c^{2}+d\right) d^{4 j}, \quad \omega_{8 j+3} P(3,4 l+2)=\left(\begin{array}{l}
l \\
j
\end{array}\right) c d^{4 j+1}, \\
\omega_{8 j+4} P(3,4 l+2)=\left(\begin{array}{l}
l \\
j
\end{array}\right) d^{4 j+2}, \quad \omega_{8 j+5} P(3,4 l+2)=0 \\
\omega_{8 j+6} P(3,4 l+2)=\left(\begin{array}{c}
l \\
j
\end{array}\right) d^{4 j+3}, \quad \omega_{8 j+7} P(3,4 l+2)=\left(\begin{array}{l}
l \\
j
\end{array}\right) c d^{4 j+3} .
\end{gathered}
$$

Note that $\omega_{8 l+6} P(3,4 l+2)=0$ and $\omega_{8 l+7} P(3,4 l+2)=0$. Furthermore, by direct computations, we obtain the following table.

TABLE 1

\begin{tabular}{|l|l|l|}
\hline & $\sigma_{8 h+p}^{(1)}$ & $\sigma_{8 h+p}^{(2)}$ \\
\hline$p=0$ & $\left(\begin{array}{c}(+1 \\
h\end{array}\right)$ & $\left(\begin{array}{c}(+1 \\
h\end{array}\right) d^{4 h}+\left(\begin{array}{c}l \\
h-1\end{array}\right)(1+c+d)\left(c+d+c d+c^{3}\right) d^{4 h-3}$ \\
\hline$p=1$ & $\left(\begin{array}{c}l+1 \\
h\end{array}\right)(1+a)$ & $\left(\begin{array}{c}l+1 \\
h\end{array}\right)(1+c) d^{4 h}+\left(\begin{array}{c}l \\
h-1\end{array}\right)(1+c)^{2}(1+c+d) d^{4 h-2}$ \\
\hline$p=2$ & 0 & $\left(\begin{array}{c}l \\
h-1\end{array}\right)(1+c)(1+c+d) d^{4 h-1}$ \\
\hline$p=3$ & 0 & $\left(\begin{array}{c}l \\
h-1\end{array}\right)(1+c)(1+c+d) d^{4 h-1}$ \\
\hline$p=4$ & 0 & $\left(\begin{array}{l}l \\
h\end{array}\right)(1+c)(1+c+d) d^{4 h}$ \\
\hline$p=5$ & 0 & $\left(\begin{array}{l}l \\
h\end{array}\right)(1+c)^{2}(1+c+d) d^{4 h}+\left(\begin{array}{c}l \\
h-1\end{array}\right)\left(c^{2}+c^{3}\right)(1+c+d) d^{4 h-1}$ \\
\hline$p=6$ & 0 & $\left(\begin{array}{l}l \\
h\end{array}\right)(1+c+d)\left(d+c d+c^{2}+c^{3}\right) d^{4 h}+\left(\begin{array}{c}l \\
h-1\end{array}\right) c^{3}(1+c+d) d^{4 h-1}$ \\
\hline$p=7$ & 0 & $\left(\begin{array}{l}l \\
h\end{array}\right)(1+c+d)\left(c^{2}+d+c d\right) d^{4 h}$ \\
\hline
\end{tabular}

Next, we look at the results of $\sigma_{l}$ from the above table. For each $\sigma_{i}$ with $i \not \equiv 0,1(\bmod 8), \sigma_{l}$ possesses the property that $\sigma_{l}^{(1)}=0$ and $\sigma_{i}^{(2)}$ always contains a 
INVOLUTIONS FIXING THE DISJOINT UNION OF 3-REAL PROJECTIVE SPACE 209

factor $(1+c+d)$. Thus for any a symmetric polynomial function $f$ which can be expressed as a sum of those monomials $\sigma_{l_{1}}^{a_{1}} \sigma_{l_{2}}^{a_{2}} \cdots \sigma_{l_{r}}^{a_{r}}$ of degree less than or equal to $8 l+12$, if each monomial $\sigma_{l_{1}}^{a_{1}} \sigma_{l_{2}}^{a_{2}} \cdots \sigma_{l_{r}}^{a_{r}}$ at least contains a elementary symmetric polynomial $\sigma_{l}$ with $i \not \equiv 0,1(\bmod 8)$ as its factors, then it is easy to see that

$$
\frac{\sigma_{l_{1}}^{a_{1}} \sigma_{t_{2}}^{a_{2}} \cdots \sigma_{l_{r}}^{a_{r}}(1+y, z)}{(1+a)}[R P(3)]+\frac{\sigma_{l_{1}}^{a_{1}} \sigma_{l_{2}}^{a_{2}} \cdots \sigma_{l_{r}}^{a_{r}}(1+y, z)}{(1+c)^{2}(1+c+d)}[P(3,4 l+2)]=0
$$

and thus

$$
\frac{f(1+y, z)}{1+a}[R P(3)]+\frac{f(1+y, z)}{(1+c)^{2}(1+c+d)}[P(3,4 l+2)]=0 .
$$

For $\sigma_{l}$ with $i \equiv 0,1(\bmod 8)$, since $\sigma_{1}^{(1)}=1+a$ and $\sigma_{1}^{(2)}=1+c$, it follows that $\sigma_{8 h+1}^{(1)}+\sigma_{1}^{(1)} \sigma_{8 h}^{(1)}=0$ and

$$
\sigma_{8 h+1}^{(2)}+\sigma_{1}^{(2)} \sigma_{8 h}^{(2)}=\left(\begin{array}{c}
l \\
h-1
\end{array}\right)(1+c+d)\left(c+c^{2}+c^{3}\right) d^{4 h-3} .
$$

In particular, we see that $\sigma_{8 h+1}^{(2)}+\sigma_{1}^{(2)} \sigma_{8 h}^{(2)}$ contains the factor $(1+c+d)$. Therefore, in further discussions, it needs only to consider $\sigma_{1}$ and $\sigma_{8 h}$ with $E(h) \subset E(l+1)$. Consider any symmetric polynomial function $f^{\prime}$ which can be expressed as a sum of those monomials $\sigma_{1}^{u} \sigma_{8 h_{1}}^{v_{1}} \sigma_{8 h_{2}}^{v_{2}} \cdots \sigma_{8 h_{r}}^{v_{r}}$ of degree less than or equal to $8 l+12$ where $E\left(h_{w}\right) \subset E(l+1)$ for $w=1, \ldots, r$. For each monomial $\sigma_{1}^{u} \sigma_{8 h_{1}}^{v_{1}} \sigma_{8 h_{2}}^{v_{2}} \cdots \sigma_{8 h_{r}}^{v_{r}}$, if $u \equiv 0(\bmod 4)$ then

$$
\begin{aligned}
\frac{\sigma_{1}^{u} \sigma_{8 h_{1}}^{v_{1}} \sigma_{8 h_{2}}^{v_{2}} \cdots \sigma_{8 h_{r}}^{v_{r}}(1+y, z)}{1+a}[R P(3)]+\frac{\sigma_{1}^{u} \sigma_{8 h_{1}}^{v_{1}} \sigma_{8 h_{2}}^{v_{2}} \cdots \sigma_{8 h_{r}}^{v_{r}}(1+y, z)}{(1+c)^{2}(1+c+d)}[P(3,4 l+2)] \\
=1+\frac{d^{4} \sum_{w=1}^{r} h_{w}}{(1+c)^{2}(1+c+d)}[P(3,4 l+2)] \\
\quad+\frac{\text { lower degree's terms of containing the factor }(1+c+d)}{(1+c)^{2}(1+c+d)}[P(3,4 l+2)] \\
=1+\frac{d^{4} \sum_{w=1}^{\prime} h_{w}}{(1+c)^{2}(1+c+d)}[P(3,4 l+2)]+0 \\
=1+\left(\begin{array}{c}
2^{N+1}-1 \\
4 l+2-4 \sum_{w=1}^{r} h_{w}
\end{array}\right)\left(\begin{array}{c}
2^{M+1}+2^{N+1}-4 l+4 \sum_{w=1}^{r} h_{w}-5 \\
3
\end{array}\right) \\
=0
\end{aligned}
$$

where $2^{N}>\max \{3,4 l+2\}, 2^{M}>3$. If $u \equiv 1,2,3(\bmod 4)$, then 


$$
\begin{aligned}
\frac{\sigma_{1}^{u} \sigma_{8 h_{1}}^{v_{1}} \sigma_{8 h_{2}}^{v_{2}} \cdots \sigma_{8 h_{r}}^{v_{r}}(1+y, z)}{1+a}[R P(3)]+\frac{\sigma_{1}^{u} \sigma_{8 h_{1}}^{v_{1}} \sigma_{8 h_{2}}^{v_{2}} \cdots \sigma_{8 h_{r}}^{v_{r}}(1+y, z)}{(1+c)^{2}(1+c+d)}[P(3,4 l+2)] \\
=0+\frac{(1+c)^{u} d^{4} \sum_{w=1}^{r} h_{w}}{(1+c)^{2}(1+c+d)}[P(3,4 l+2)] \\
+\frac{(1+c)^{u}(\text { lower degree's terms of containing the factor }(1+c+d))}{(1+c)^{2}(1+c+d)} \\
\quad \times[P(3,4 l+2)] \\
=\frac{(1+c)^{u} d^{4} \sum_{w=1}^{r} h_{w}}{(1+c)^{2}(1+c+d)}[P(3,4 l+2)]+0 \\
=\left(\begin{array}{c}
2^{N+1}-1 \\
4 l+2-4 \sum_{w=1}^{r} h_{w}
\end{array}\right)\left(\begin{array}{c}
2^{M+1}+2^{N+1}-4 l+4 \sum_{w=1}^{r} h_{w}-5+u \\
3
\end{array}\right) \\
=0 . \quad(\text { since } u \equiv 1,2,3(\bmod 4))
\end{aligned}
$$

So we can still obtain that

$$
\frac{f^{\prime}(1+y, z)}{1+a}[R P(3)]+\frac{f^{\prime}(1+y, z)}{(1+c)^{2}(1+c+d)}[P(3,4 l+2)]=0 .
$$

Combining the above discussions, we complete the proof.

Proof of Theorem 5.1 (II). First, it is easy to see that for $2 \leq k \leq 4 A-3$, the vector bundle $\quad \imath \oplus(8 l+k-1) R \rightarrow R P(3) \sqcup \eta \oplus(k-2) R \rightarrow P(3,4 l) \quad$ is bordant to $v_{1}^{8 l+k} \rightarrow R P(3) \cup v_{2}^{k} \rightarrow P(3,4 l)$ with $\omega v_{1}=1+a$ and $\omega v_{2}=1+c+d$ where $R$ denotes the trivial line bundle. Hence by Corollary 3.3, we need merely to check the case $k=4 A-3$ of Theorem 5.1 (II). Let $k=4 A-3$. Now, by means of Proposition 3.2, we show that $v_{1} \rightarrow R P(3) \sqcup v_{2} \rightarrow P(3,4 l)$ with $\omega v_{1}=$ $1+a$ and $\omega v_{2}=1+c+d$ is the fixed point data of involution $\left(T, M^{8 l+4 A}\right)$. We proceed as follows:

STEP (i). The computation of $\sigma_{l}$ for $0 \leq i \leq 8 l+4 A$. Using [5; p. 317, Lemma], we have

$$
\sigma_{\imath}^{(1)}=\left(\begin{array}{c}
8 l+4 A-3 \\
i
\end{array}\right)+\left(\begin{array}{c}
8 l+4 A-4 \\
i-1
\end{array}\right) a
$$

and

$$
\sigma_{l}^{(2)}=\sum_{q \leq l}\left(\begin{array}{c}
4 A-3 \\
i-q
\end{array}\right) \sigma_{q}(z)+c \sum_{1+q \leq l}\left(\begin{array}{c}
4 A-4 \\
i-q-1
\end{array}\right) \sigma_{q}(z)+d \sum_{2+q \leq l}\left(\begin{array}{c}
4 A-5 \\
i-q-2
\end{array}\right) \sigma_{q}(z) .
$$

From

$$
\omega P(3,4 l)=(1+c)^{3}(1+c+d)^{4 l+1},
$$


it follows that

$$
\begin{aligned}
& \omega_{2 j} P(3,4 l)=d^{j}, \quad \omega_{2 j+2} P(3,4 l)=d^{J+1}, \quad \omega_{2 j+3} P(3,4 l)=c d^{J+1}, \\
& \omega_{2 j+4} P(3,4 l)=c^{2} d^{J+1}, \quad \omega_{2 j+5} P(3,4 l)=c^{3} d^{J+1}, \quad \omega_{h} P(3,4 l)=0
\end{aligned}
$$

where $E(j) \subset E(4 l), h \neq 2 j, 2 j+2,2 j+3,2 j+4,2 j+5$.

Let $E(r) \subset E(4 l)$. First, we compute $\sigma_{2 r+p}$ for $p \leq 4 A-1$. The results are stated as follows.

TABLE 2

\begin{tabular}{|l|l|l|}
\hline & $\sigma_{2 r+p}^{(1)}$ & $\sigma_{2 r+p}^{(2)}$ \\
\hline$p=8 H$ & 1 & $\begin{array}{l}d^{r}\left(1+c(1+c)^{3} d(1+c+d)\right), \text { if } H \neq 0 ; d^{r}, \text { if } H=0 \text { and } \\
E(2 r-4 A) \not \subset E(8 l) ; d^{r}+c d^{r-2 A+1}(1+c)^{3}(1+c+d), \text { if } H=0 \text { and } \\
E(2 r-4 A) \subset E(8 l) .\end{array}$ \\
\hline$p=8 H+1$ & $1+a$ & $\begin{array}{l}d^{r}\left(1+c+c^{2} d+c^{2} d^{2}+c^{3} d^{2}\right), \text { if } H \neq 0 ; d^{r}(1+c), \text { if } H=0 \text { and } \\
E(2 r-4 A) \not E(8 l) ;(1+c) d^{r}+c^{2} d^{2 r-2 A+1}(1+d+c d), \text { if } H=0 \text { and } \\
\end{array}$ \\
\hline$p=8 H+2$ & 0 & $\begin{array}{l}d^{r}\left(c^{3} d+c^{3} d^{2}\right), \text { if } H \neq 0 ; 0, \text { if } H=0 \text { and } E(2 r-4 A) \not \subset E(8 l) ; \\
3^{3} d^{r-2 A+1}+c^{3} d^{r-2 A+2}, \text { if } H=0 \text { and } E(2 r-4 A) \subset E(8 l) .\end{array}$ \\
\hline$p=8 H+3$ & 0 & 0 \\
\hline$p=8 H+4$ & 1 & $d^{r}\left(1+c d+d+d^{2}\right)$ \\
\hline$p=8 H+5$ & $1+a$ & $d^{r}\left(1+c+d+d^{2}+c^{2} d+c d^{2}\right)$ \\
\hline$p=8 H+6$ & 0 & $d^{r}\left(d+c^{3} d+d^{2}+c d^{2}+c^{2} d^{2}\right)$ \\
\hline$p=8 H+7$ & 0 & $d^{r}\left(d+d^{2}+c d^{2}+c^{2} d^{2}+c^{3} d^{2}\right)$ \\
\hline
\end{tabular}

Next, we compute $\sigma_{2 r+p}$ for $p \geq 4 A$. Let

$$
u=\min \{w \mid E(2 r+4 A w) \not \subset E(8 l) \text { and } w \geq 1\} .
$$

When $4 A \leq p \leq 4 A u-1(u>1)$, since $E(2 r+4 A w) \subset E(8 l)$ for each $w \leq u-1$, thus the computation of $\sigma_{2 r+p}$ for $4 A w \leq p \leq 4 A-1$ can be completely iterated as the computation procedure of the case $0 \leq p \leq 4 A-1$, and results can be obtain as long as $r$ in the results of Table 2 is replaced by $r+2 A w$.

When $p \geq 4 A u$, if $2 r+4 A u=8 l+4 A$, we have $\sigma_{8 l+4 A}=0$. If $2 r+4 A u<$ $8 l+4 A$, let $v=\min E(2 r+4 A u)$, then we have

$$
\sigma_{2 r+4 A u}^{(1)}=0, \quad \sigma_{2 r+4 A u}^{(2)}= \begin{cases}d^{r}\left(c d+c d^{2}+c^{2} d^{2}+c^{3} d^{2}\right), & \text { if } u=1 \\ 0, & \text { if } u>1\end{cases}
$$




$$
\begin{gathered}
\sigma_{2 r+4 A u+1}^{(1)}=0, \quad \sigma_{2 r+4 A u+1}^{(2)}= \begin{cases}d^{r}\left(c^{2} d+c^{2} d^{2}+c^{3} d^{2}\right), & \text { if } u=1 ; \\
0, & \text { if } u>1 .\end{cases} \\
\sigma_{2 r+4 A u+2}^{(1)}=0, \quad \sigma_{2 r+4 A u+2}^{(2)}= \begin{cases}d^{r}\left(c^{3} d+c^{3} d^{2}\right), & \text { if } u=1 ; \\
0, & \text { if } u>1 .\end{cases} \\
\sigma_{2 r+4 A u+I}=0, \quad \text { if } 3 \leq I \leq 2^{v}-1 .
\end{gathered}
$$

In the next computations, when $p$ exceeds $4 A u+2^{v}-1$, since either $E\left(2 r+4 A u+2^{v}\right) \subset E(8 l)$ or $E\left(2 r+4 A u+2^{v}\right) \not \subset E(8 l)$ always holds, hence whichever of both happens, we always can iterate the above computation procedure. In particular, the results of all $\sigma_{l} \neq 0$ must belong to those forms showed in table 2, (5.1), (5.2), (5.3).

STEP (ii). From the computation results of all $\sigma_{l}(i=0,1, \ldots, 8 l+4 A)$, it is easy to see that only using $\sigma_{0}, \sigma_{1}, \sigma_{4}, \sigma_{4 A}$, each $\sigma_{i}(i \neq 0,1,4,4 A)$ having the property $\sigma_{\imath} \neq 0$ can be changed into $\sigma_{l}^{\prime}$ of degree $i$ such that $\sigma_{l}^{\prime}=0$. This means that for any symmetric polynomial function $f(x)$ of degree less than or equal to $8 l+4 A, f$ can be generated by $\sigma_{0}, \sigma_{1}, \sigma_{4}, \sigma_{4 A}$. In other words, $f$ can be expressed as the sum of those expressions $\sigma_{0}^{l_{1}} \sigma_{1}^{l_{2}} \sigma_{4}^{l_{3}} \sigma_{4 A}^{l_{4}}$. Furthermore, we can easily obtain that for any symmetric polynomial function $f(x)$ of degree less than $8 l+4 A$,

$$
\frac{f(1+y, z)}{1+a}[R P(3)]+\frac{f(1+y, z)}{1+c+d}[P(3,4 l)]=0
$$

and thus $v_{1} \rightarrow R P(3) \sqcup v_{2} \rightarrow P(3,4 l)$ with $\omega v_{1}=1+a$ and $\omega v_{2}=1+c+d$ is the fixed point data of $\left(T, M^{8 l+4 A}\right)$ by Proposition 3.2. This completes the proof.

\section{§6. The representative of involution up to bordism}

In this section, we discuss the representatives up to bordism of those involutions stated in Theorem 5.1.

Lemma 6.1. There exists an involution $G$ on $P(3, n+1)$ such that the fixed point set of $(G, P(3, n+1))$ is $R P(3) \sqcup P(3, n)$.

Proof. First, setting an involution $g$ on $S^{3} \times C P(n+1)$ by

$$
g:\left(\left(x_{0}, x_{1}, x_{2}, x_{3}\right),\left(z_{0}, z_{1}, \ldots, z_{n}\right)\right) \rightarrow\left(\left(x_{0}, x_{1}, x_{2}, x_{3}\right),\left(-z_{0}, z_{1}, \ldots, z_{n}\right)\right) .
$$

It is obvious that the fixed point set of $\left(g, S^{3} \times C P(n+1)\right)$ is $S^{3} \times C P(0) \sqcup$ $S^{3} \times C P(n)$. Next, we can at once obtain an involution $G$ on $P(3, n+1)$ induced by $\left(g, S^{3} \times C P(n+1)\right)$, and easily see that the fixed point set of $(G, P(3, n+1))$ is exactly $R P(3) \sqcup P(3, n)$. This completes the proof.

By the Theorem 5.1, Remark 4.1 and Lemma 3.5, it immediately follows that 
THEOREM 6.2. (i) The involution $\left(T, M^{3+8 l+k}\right)$ stated in Theorem 5.1 (II) is bordant to $\left(G_{k-2}, \Gamma^{k-2}(P(3,4 l+1))\right)$ where $2 \leq k \leq 4 A-3$.

(ii) The involution $\left(T, M^{7+8 l+k}\right)$ stated in Theorem 5.1 (III) is bordant to $\left(G_{k-2}, \Gamma^{k-2}(P(3,4 l+3))\right)$ where $2 \leq k \leq 4$.

As for the representatives up to bordism of those involutions stated in Theorem 5.1 (I), (IV), we have done many tries, but nothing conclusive.

\section{REFERENCES}

[1] P E. ConNer AND E. E. Floyd, Fiberıng withın a cobordism class, Michigan Math. J., 12 (1965), 33-47

[2] P E. Conner, Differentiable Perıodic Maps, 2nd ed., Lecture Notes in Math., 738 Springer, Berlin, 1979.

[3] M. FujII AND T. YASUI, KO-cohomologies of Dold manifolds, Math. J. Okayama Unıv., 16 (1973), 55-84.

[4] D. Hou AND B. F. Torrence, Involutions fixing the disjoint union of odd-dimensional projective spaces, Canad. Math. Bull., 37 (1994), 66-74.

[5] C. Kosniowski AND R. E. STONG, Involutions and characteristic numbers, Topology 17 (1978), 309-330.

[6] Z. LÜ AND C. L. ZHou, A note on the boardman theorem, Internat. J. Math. Math. Sc1., 21 (1998), 819-822.

[7] J. W Milnor and J. D. StashefF, Characterıstıc Classes, Princeton University Press, Princeton, 1974.

[8] Pedro L. Q. Pergher, Involutions fixing an arbitrary product of spheres and a point, Manuscripta Math., 89 (1996), 471-474.

[9] D. C. Royster, Involutions fixıng the disjoint unıon of two projectıve spaces, Indiana Unıv. Math. J. 29 (1980), 267-276.

[10] D. Singmaster, Notes on binomial coefficients, J. London Math. Soc., 8 (1974), 545-560.

[11] R. E. Stong, Involutions fixıng the projectıve spaces, Michigan Math. J., 13 (1966), 445-447

[12] R. E. Stong, Involutions fixıng products of circles, Proc. Amer. Math. Soc., 119 (1993), 1005-1008.

[13] B. F TORRENCE, Bordism classes of vector bundles over real projective spaces, Proc. Amer. Math. Soc., 118 (1993), 963-969.

[14] J. J. UCCI, Immersions and embeddings of Dold manifolds, Topology, 4 (1965), 283-293.

Graduate School of Mathematical Sciences

THE UNIVERSITY OF TOKYO

3-8-1 KOMABA, TOKYO 153-8914, JAPAN

DePartment of APPlied Mathematics

TSINGHUA UNIVERSITY

BeIJing, 100084, P. R. China

e-mail: zlu@ms326yzk.ms.u-tokyo.ac.jp

INSTITUTE OF SySTEMS SCIENCE

ACADEMIa Sinica

BeIJing, 100080, P R. China

Hebei University of Science and Technology

ShiJiazhuang, 050054, P R. China 